\title{
Human Factors for Capacity Building. Lessons learned from the OpenMRS Implementers Network
}

\author{
C. J. Seebregts $s^{a, b}$, B. W. Mamlini, , P. G. Biondich, , , H. S. F. Frasere, , B. A. Wolfe, D. Jazayerif, \\ J. Miranda $a^{f}$ J. Blaya a, , C. Sinha ${ }^{h}$, C. T. Bailey', A. S. Kanteri \\ a Biomedical Informatics Research, eHealth Research and Innovation Plafform, Medical Research \\ Council, Cape Town, South Africa \\ b School of Computer Science, University of KwaZulu-Natal, Durban, South Africa \\ ' Regenstrief Institute, Indianapolis, USA \\ d Indiana University School of Medicine, Indianapolis, USA \\ • Brigham and Women's Hospital, Boston, USA \\ 'Partners in Health, Boston, USA \\ ${ }^{9}$ eHealth Systems, Santiago, Chile \\ h International Development Research Centre (IDRC), Ottawa, Canada \\ ' eHealth and Informatics Unit, Knowledge Management and Sharing Department, World Health \\ Organization, Geneva, Switzerland \\ i Millennium Villages Project, Earth Institute, Columbia University, New York, USA
}

\begin{abstract}
Summary
Objectives: The overall objective of this project was to investigate ways to strengthen the OpenMRS community by (i) developing capacity and implementing a networkfocusing specifically on the needs of OpenMRS implementers, (ii) strengthening community-driven aspects of OpenMRS and providing a dedicated forum for implementation-specific issues, and; (iii) providing regional supportfor OpenMRS implementations as well as mentorship and training.

Methods: The methods used induded (i) face-to-face networking using meetings and workshops; (ii) online collaboration tools, peer support and mentorship programmes; (iii) capacity and communitydevelopment programmes, and; (iv) community outreach programmes.

Results: The community-driven approach, combined with a fewsimple interventions, has been a key factor in the growth and success of the OpenMRS Implementers Network. It thas contributed to implementations in at least twenty-three different countries using basic online tools; and provided mentorship and peer support through an annual meeting, workshops and an internship program. The OpenMRS Implementers Network has formed collaborations with several other open source networks and is sevolving regional OpenMRS Centres of Excellence to provide localized support for OpenMRS development and implementation. These initiatives are increasing the range offunctionality and sustainability of open source software in the health domain, resulting in improved adoption and enterprise-readiness.

Conclusions: Social organization and capacitydevelopment activities are important in growing a successful community-driven open source software model.

\section{Keywords}

Medical record systems, computerized patient records, free and open source software, medical informatics

Yearb Med Inform 2010:13-20
\end{abstract}

\section{Introduction}

Appropriate use of information using a structured electronic medical record can lead to better decisions that can, in turn, lead to improved patient care and public health management $[1,2]$. There are several electronic medical record systems currently deployed in low-resource developing country settings. Many of these are bespoke systems that require ongoing and costly maintenance and support [3, 4]. Electronic medical record systems designed using free and open source software (FOSS) principles and approaches allow for greater flexibility, engagement and feedback from the implementers and users of the systems where they are being deployed. This article presents one such system, OpenMRS, and how the human factors - notably social organization and capacity development approaches and tools - have influenced its trajectory, membership and outcomes, to date. The remainder of the article is structured to: provide an overview of OpenMRS and its different component activities; a list of objectives set out for the OpenMRS Implementers Network (OIN); a summary of results; and some concluding thoughts on progress to date and what lies ahead.

\section{Overview of the OpenMRS Implementers Network (OIN) OpenMRS}

The Open Medical Record System (OpenMRS) is a configurable, open source electronic medical record application, developed and maintained jointly by the Regenstrief Institute (RI) at Indiana University and Partners in Health (PIH) in collaboration with a large group of volunteer, open source developers from around the world $[5,6,7]$. The core OpenMRS developer group, comprising six developers is funded by grants from international donors, including the Centres for Disease Control and the Rockefeller Foundation. The OpenMRS source code and compiled distributions of the core application are freely and openly available from the OpenMRS web site (www.openmrs.org) under the terms of the OpenMRS Public License, a derivative of the Mozilla Public License. The core OpenMRS web application provides basic patient and clinical encounter management functionality and a configurable set of observations linked to well-defined concepts. Additional functionality can be added to the core application by installing optional modules. Although developed with 
complicated HIV and TB care in mind, OpenMRS is primarily focused on the needs of small primary health care facilities in developing countries. It includes a robust, yet configurable backend application and MySQL database, supplemented with HTML-forms, front-end functionality to capture data from typical paper encounter forms. An improved graphical user interface with richer functionality to support additional needs of clinicians is currently under development.

\section{The OpenMRS Implementers Network}

The OpenMRS Implementers Network (OIN) was initiated by the OpenMRS Leadership in February 2006 following an initial training session for OpenMRS implementers at the AMPATH (Academic Model for Providing Access to Healthcare) Program in Eldoret, Kenya. OpenMRS distinguishes between implementers and developers. OpenMRS Implementers are technical non-programmers who are responsible for configuring and implementing OpenMRS, but do not necessarily contribute to the development. OpenMRS developers contribute code to OpenMRS, either in the core application or modules and may also act as Implementers. The original vision of the OIN was to grow the community aspects of OpenMRS. The main aims of the OIN are to provide a dedicated forum for OpenMRS implementers and implementation-specific issues, provide regional support for OpenMRS implementations and provide mentorship and training for non-core developers and implementers, predominantly in Africa and other low-resource settings.

The first actions of the OIN were to organize the inaugural OpenMRS Implementers meeting in Cape Town during June 2006, followed by the first implementer-driven implementation of a standard OpenMRS application at Richmond Chest Hospital in KwaZulu-Natal, South Africa, by the South African Medical Research Council (MRC) as part of the Open Architectures, Standards and Information Systems (OASIS) project, funded by the International Development
Research Centre (IDRC). Since then, the OIN has grown into a worldwide consortium of implementers and developers, developing functionality and supporting implementations around the world [9]. Moreover, the community is not limited to those who use OpenMRS technology, but rather it is a self-selecting group that shares core values of knowledge sharing and mutual support.

The OpenMRS core group and central developer team supports the OIN mostly by way of a dedicated list server and an annual meeting in Africa that brings representatives of the developer and implementer communities together. Moreover, OpenMRS has developed capacity building programs within the OIN including the OpenMRS Internship program and an OpenMRS training program associated with the implementation of OpenMRS at primary health facilities in Rwanda $[10,11]$. More recently, OpenMRS has also begun to recognize OpenMRS Centres of Excellence, including the Millennium Villages Project (MVP) [12], Jembi Health Systems, a South African NGO and eHealth Systems (eHS), a private company regis- tered in Chile. The group also recognizes reference implementations that have development capacity, including the original OpenMRS implementations at AMPATH in Eldoret, Kenya and PIH in Rwinkwavu, Rwanda as well as implementations by the Health Information Systems Program (HISP) in India, Interactive Research and Development (IRD) in Pakistan and the BAOBAB project in Malawi [13] that uses a version of the OpenMRS data model. The different components of the OIN can be loosely visualized as shown below in Figure 1. There is an overlap between these components, which illustrates the seamless ways in which the components unfold. The parts of Figure 1 are discussed in the following sections, and then revisited in the section on results.

\section{Objectives}

The objectives of the OIN guide its current activities and approaches, and inform future strategic direction to continue providing support to the

\section{OpenMRS Implementers Network (OIN)}

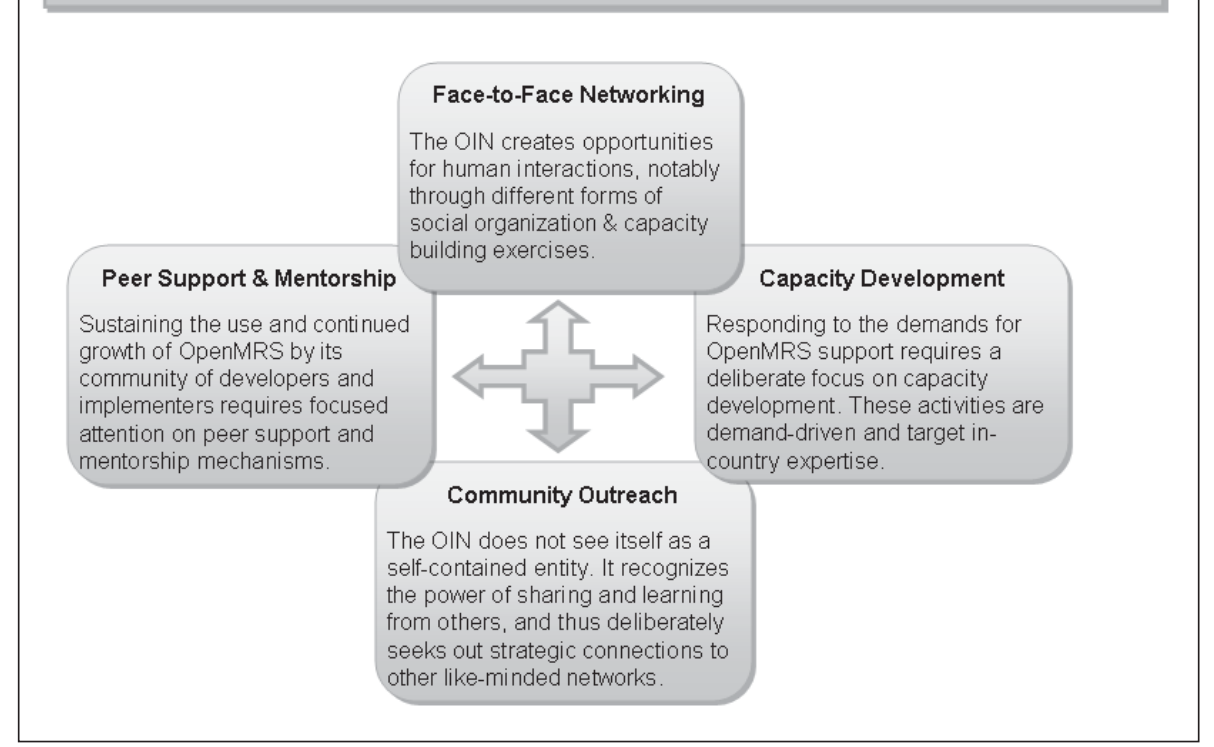

Fig. 1 Components of the OpenMRS Implementers Network 
community of interest. These objectives are summarized below:

- To build capacity for OpenMRS development and implementation in developing countries.

- To implement a network focusing specifically on the needs of OpenMRS implementers.

- To strengthen community-driven aspects of OpenMRS.

- To provide a dedicated forum for implementation-specific issues.

- To provide regional support for OpenMRS implementations in Africa

- To provide mentorship and training for African developers and implementers.

\section{Methods}

Different activities and approaches have been implemented to help achieve the objectives of the OIN, and to measure the extent of their achievement.

\section{Face-to-Face Networking}

\section{OIN Annual Meetings and Workshops \\ OIN Annual Meeting}

The OpenMRS Implementers Network Meeting is organised annually in Africa by the South African Medical Research Council (MRC) and Jembi, and funded in part by Canada's IDRC. Travel funding is provided to a core group of OpenMRS developers and selected implementers from low-resource countries, mostly in Africa and South East Asia.

\section{Workshops}

The OIN has held several workshops in Africa, both as part of the annual Implementers Meeting and also independently. The 2008 OIN Annual Meeting had a dedicated facility for OpenMRS and other free and open source (FOSS) developers attending the meeting and focusing on inter-operability. An OpenMRS group training session was held before the 2009 OIN Annual Meeting for OpenMRS developer leads with developers from the OASIS project and other developers from lowresource settings. The OASIS project has held corresponding OpenMRS workshops in South Africa, Mozambique and Zimbabwe and PIH held a one-day OpenMRS workshop in Boston during May 2009. In addition, the Sustainable Sciences Institute and eHealth Systems (eHS) have started holding workshops in Latin America, the first of which was in Peru in 2009. The workshops are notable for their emphasis on peer-to-peer learning techniques rather than more traditional presentation/Q\&A formats.

\section{Peer Support and Mentorship \\ The OpenMRS Implementers Network and Online Collaboration Tools}

The OpenMRS Wiki (MediaWiki) is the most important and powerful online collaboration tool available to the OpenMRS developer and implementer communities. OpenMRS also operates several dedicated list servers associated with different constituencies within the community. The OIN has a dedicated list server (OPENMRS-IMPLEMENTERS) that is the predominant means of collaboration and communication among implementers. It is also the primary way in which support is delivered to implementers either by other implementers or by developers.

\section{OpenMRS Internship Programme}

The OpenMRS Internship Programme (OIP) is modelled on the Google Summer of Code (GsoC; http://socghop. appspot.com/) and applied to developing countries. OpenMRS was selected to participate in GSoC for the first time in 2007 and since then has participated annually. The following general process is followed: OpenMRS community post projects; applicants submit a proposal based on the project or a new idea; OpenMRS mentor community scores the projects and applies for support from Google. Based on the funding provided by Google, successful projects are selected. Interns are matched with a primary and secondary mentor. Interns are required to submit a project plan that is approved and need to submit progress reports in order to receive the bulk of the stipend. OIP Interns follow exactly the same format as $\mathrm{GSoC}$ interns but must either originate from a developing country or be living and working in a developing country in order to be eligible.

\section{Capacity Development}

Open Architectures, Standards and Information Systems (OASIS) Project The OASIS project has developed nodes in three African countries: South Africa, Mozambique and Zimbabwe. Week-long training programs were developed for these nodes and include a combination of theoretical training in principles of patient management systems development followed by practical training in OpenMRS. Special emphasis has been place on using Enterprise Architecture approaches to encourage national dialogues around planning of national health information systems based on actual information flows and needs in a software agnostic way.

\section{EHSDI - OpenMRS and Java Training program in Rwanda}

The EHSDI - OpenMRS and Java Training program in Rwanda is a capacity development initiative initiated by PIH and funded by IDRC, in which interns in Rwanda are trained for one year in basic and advanced Java and OpenMRS development [14]. The original intention was to provide capacity to assist with the government-requested rollout of OpenMRS in Rwanda [10]. The students receive a stipend from the Rwandan Information Technology Administration during their training and are then contracted to work with the Ministry of Health afterwards for 1-2 years. Based on a three month pilot training program, the course was extended to nine months to give sufficient time to cover all the necessary technologies. 
Moreover, a thorough screening of applicants was put in place to ensure that only high quality students were recruited, and the involvement of a sufficient number of mentors was recommended to ensure all students received a practical, "hands on" experience. It was agreed that there should be no more than 6 students per mentor. The course involves the teaching of core medical informatics principles, and exposure to the real issues on the ground through field trips as well as visits from staff implementing and using the systems. All course materials and experiences are being documented to be shared by other organizations, and the students should be evaluated at regular stages throughout the year.

Based on the strengths and weaknesses of the students shown in the screening exams a curriculum was created with the following seven topics: (i) Foundations; (ii) Basic Web Design; (iii) Basic Java Programming; (iv) Advanced Java Programming; (v) OpenMRS Implementation; (v) Enterprise Java Programming; (vi) OpenMRS Development, and; (vii) Medical Informatics.

\section{Reference Implementations and Centres of Excellence}

In 2009, the OpenMRS Leadership Group decided to formally acknowledge OpenMRS Reference Implementations. The main intention of these sites is to serve as a practical basis for feature prioritization and "on-the-ground" requirements gathering. These reference sites, in other words, help lead the software development roadmap process. Criteria for recognition as a reference implementation include sustained implementation of OpenMRS at a single site over a period of time, and a demonstrated contribution to further growth of the OpenMRS community, both from a capacity development and a software development perspective.

\section{Community Outreach}

As a community, OpenMRS has reached out to a number of other open source health software development com- munities. At this stage, the most significant collaborations are with the World Health Organization and the development of SDMX-HD (www.sdmx-hd.org), a standard for aggregate data management and the University of Oslo and Health information Systems Program (HISP; www.hisp.org), developers of the District Health Information System (DHIS) $[15,16]$ software and OpenROSA (www.openrosa.org), an open source community that develops and maintains JavaROSA, a mobile phone data collection application. Developers from CellLife and Jembi have also integrated OpenMRS with integrated with iDart (www.cell-life.org/idart), an HIV pharmacy and dispensary management application, also maintained by Cell-Life.

\section{Results}

\section{Face-to-Face Networking}

OpenMRS Implementers Network Annual Meeting and Workshops

The annual OpenMRS Implementers Meeting began with 67 attendees in 2006 and increased to 167 in 2007. Approximately 180 attendees attended the 2008 meeting that was held in conjunction with HISA (Health Informatics in Southern Africa) 2008, the biannual conference of the South African Health Informatics Association (SAHIA). In 2009 , the meeting was restricted to 138 attendees for logistical reasons and more than a hundred other applicants could not be accommodated.

In 2006, the OIN Meeting was structured as a conventional conference with keynote presentations and some unstructured interactive sessions. In response to a requirement for greater attendee participation, since 2007-the format of the OIN Meeting has changed to place a stronger emphasis on discussion sessions and a dynamic agenda. By 2009, the OIN Meeting had adopted a complete un-conferencing format in which the first item on the agenda was to set the agenda. The meeting comprises a minimal number of standard presentations and is mostly made up of sessions requested by the attendees. The format of the sessions includes keynote presentations on selected topics (e.g., State of OpenMRS), discussion fora, fishbowls and technical workshops and is matched to the type of content and participants. In recent years, the OIN Meeting has also increased the technical content with an emphasis on bidirectional interaction between developers and implementers. Attendees to the OIN Meeting include a roughly equal number of implementers and developers.

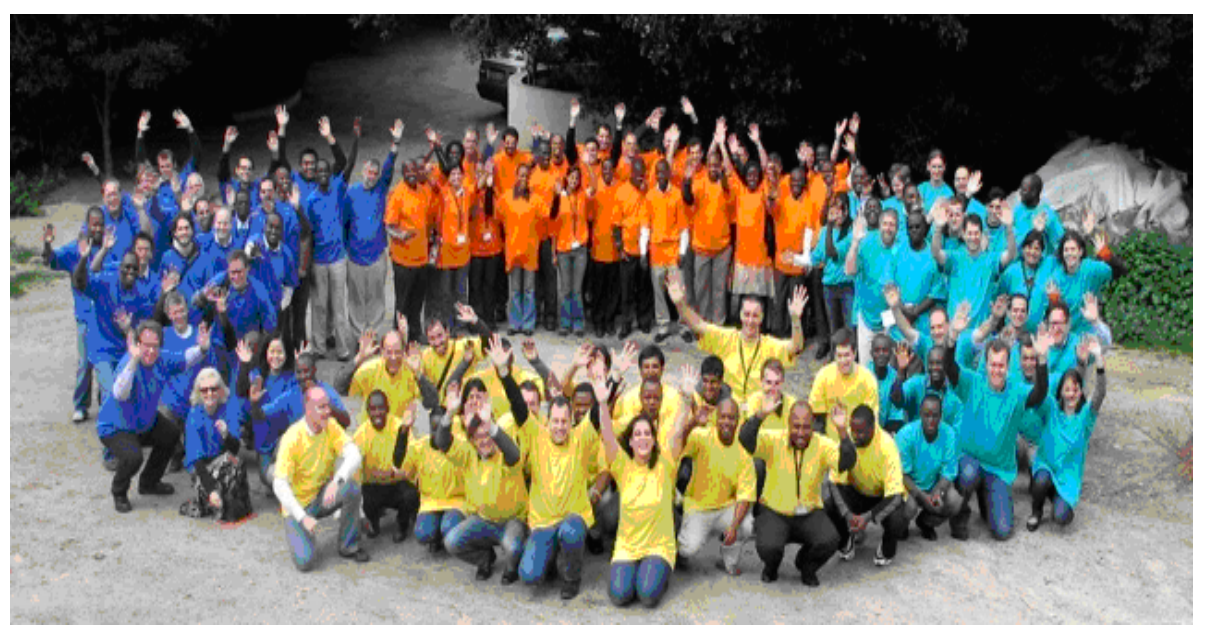

Fig. 2 Group Photograph of Attendees to the 2009 OpenMRS Implementers Network Annual Meeting. 


\section{Peer Support and Mentorship}

The OpenMRS Implementers Network and Online Collaboration Tools

The OpenMRS Implementers Network has grown from a simple idea in 2006 to a worldwide network of implementers and implementations. It is difficult to obtain an accurate estimate of the number of implementations and geographic spread of the OIN on account of the fact that OpenMRS is freely downloadable from the OpenMRS web site. However, estimates can be obtained, indirectly, from other indicators. Thirty-three implementations of OpenMRS in twenty-three different countries are known to the OpenMRS Leadership Group, as shown in the map in Figure 3. This does not include test and pilot implementations. It is likely that the actual number of OpenMRS implementations is significantly higher since querying the OpenMRS list server logs shows that OpenMRS has been downloaded by 10,589 individual IP addresses from 88 different countries (Table 1). This an indicator of the extent of the interest. However, it does not take into account multiple downloads from the same person or organization and will also likely include a large number of casual browsers who do not go on to implement OpenMRS in a live setting.

\section{Online Collaboration Tools}

The OpenMRS Implementers list has registered 244 implementers and 256 developers since 7 April 2008 (when recording began). A total of 386 unique names are registered across both lists (i.e., 114 people appear on both lists). 126 committers have contributed code to both core and modules to date.

\section{OpenMRS Internship Program (OIP)/} Google Summer of Code (GSoC)

OpenMRS has participated in the GSoC programme for the past three years and, in 2010, had one of the highest number of interns funded by Google of any open source project. In 2007,

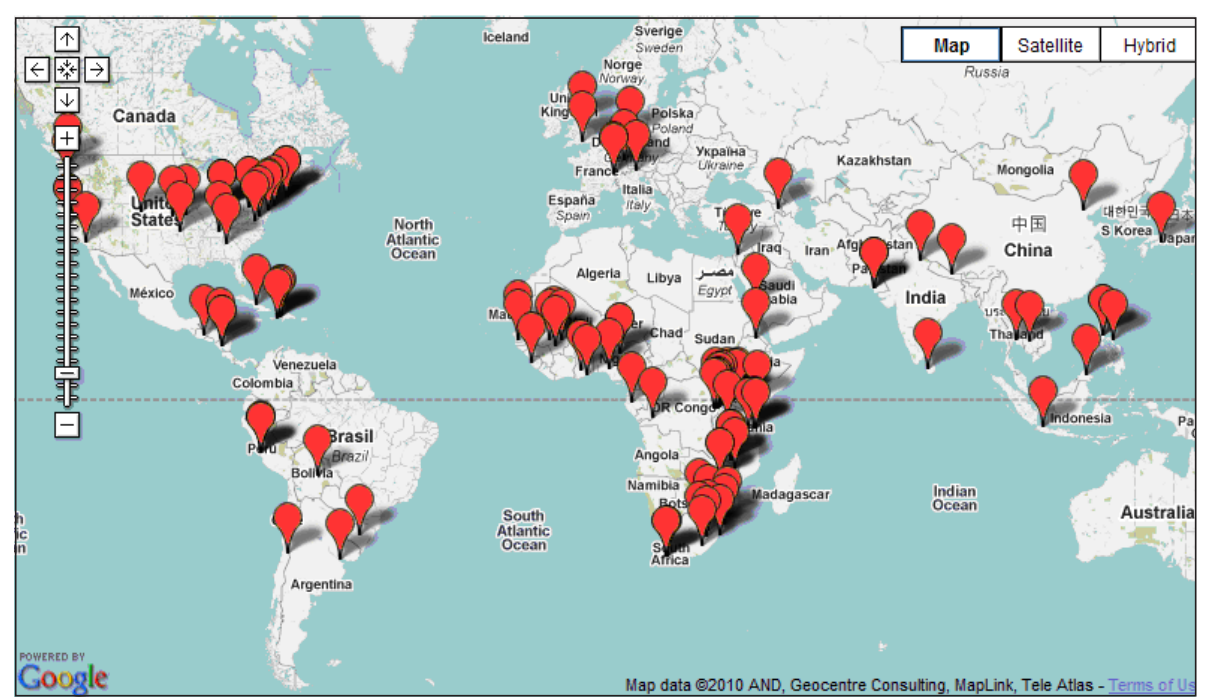

Fig. 3 Geographical Distribution of Known OpenMRS Implementations

12 interns successfully completed the OpenMRS-GSoC program, 9 interns completed the program in 2008, 16 in 2009 and 17 in 2010 and a significant number of modules and other core functionality has been developed as a result of this program. The OIP has been running since 2008. Eight interns were accepted in the first year of which three completed the program. Two OIP interns have been accepted in the second year and one completed the program. Two interns have been accepted in 2010 .

\section{Capacity Development}

\section{OASIS Training Programs}

Four-day training programs for OpenMRS were held in Zimbabwe during 2008 and one training program was held in Mozambique by the OASIS project, and have involved between 5 and 20 participants.

\section{EHSDI - OpenMRS and Java Training program in Rwanda}

The EHSDI course was run, successfully, in 2008-2009. The course was 11 months in duration in the first year reduced to nine in year two. It comprises an initial three months of basic coursework, that also serves as a probation period, and includes two months of project work developing a module for OpenMRS. Candidates were selected by a written screening exam based on understanding of basic IT concepts, the software development cycle, construction of simple algorithms and awareness of more advanced topics like object orientated programming. They were found to be generally familiar with theoretical IT concepts but weak on creating even simple algorithms. Nineteen students were interviewed and ten were selected for the course. All 10 students graduating in October 2009 in a ceremony attended by the Rwandan Permanent Secretary of health. All students successfully took part in the development of modules that are now being used in Rwanda by PIH or the MOH. Eight are now working on OpenMRS or similar systems and a graduate from the first year of training is now assisting to teach the course. After receiving 160 initial applicants, twelve students were enrolled in the second year program in February 2010 .

\section{Centres of Excellence}

Three Centres of Excellence in OpenMRS development and implementation have developed outside of the original two hubs at the Regenstrief In- 
Table 1 Country Downloads of OpenMRS

\begin{tabular}{|c|c|c|c|}
\hline Country & Count & Country & Count \\
\hline United States & 5623 & Oman & 13 \\
\hline India & 1118 & Slovenia & 13 \\
\hline United Kingdom & 430 & Bulgaria & 12 \\
\hline Indonesia & 221 & New Zealand & 12 \\
\hline Italy & 220 & Hungary & 10 \\
\hline Pakistan & 208 & Malta & 10 \\
\hline Philippines & 202 & Nigeria & 9 \\
\hline Russian Federation & 190 & Republic of Korea & 9 \\
\hline China & 186 & Cambodia & 8 \\
\hline Sri Lanka & 169 & Monaco & 8 \\
\hline Germany & 165 & Republic of Moldova & 6 \\
\hline Canada & 147 & Singapore & 6 \\
\hline Uganda & 135 & Estonia & 5 \\
\hline Spain & 123 & Kazakhstan & 5 \\
\hline Australia & 97 & Lebanon & 5 \\
\hline France & 86 & Nepal & 5 \\
\hline Kenya & 82 & Jordan & 4 \\
\hline Malaysia & 77 & Kuwait & 4 \\
\hline Netherlands & 77 & Yemen & 4 \\
\hline Saudi Arabia & 73 & Croatia & 3 \\
\hline Greece & 72 & Denmark & 3 \\
\hline Taiwan & 59 & Ghana & 3 \\
\hline Vietnam & 56 & Hong Kong & 3 \\
\hline Portugal & 49 & Japan & 3 \\
\hline Norway & 46 & Former Yugoslav Republic of & 3 \\
\hline Romania & 42 & Macedonia & \\
\hline Switzerland & 42 & United Arab Emirates & 3 \\
\hline Thailand & 35 & Bahrain & 2 \\
\hline Israel & 31 & Bosnia and Herzegovina & 2 \\
\hline Syrian Arab Republic & 31 & Brunei Darussalam & 2 \\
\hline Puerto Rico & 30 & Cyprus & 2 \\
\hline Bangladesh & 29 & Jamaica & 2 \\
\hline Sweden & 28 & Latvia & 2 \\
\hline Ukraine & 26 & Lithuania & 2 \\
\hline Turkey & 25 & Nicaragua & 2 \\
\hline South Africa & 24 & Serbia and Montenegro & 2 \\
\hline Poland & 21 & Afghanistan & 1 \\
\hline Ireland & 18 & Chile & 1 \\
\hline Niger & 16 & Czech Republic & 1 \\
\hline Austria & 15 & Luxembourg & 1 \\
\hline Honduras & 15 & Palestinian Territory, Occupied & 1 \\
\hline Finland & 14 & Qatar & 1 \\
\hline Gambia & 14 & Sierra Leone & 1 \\
\hline Belgium & 13 & Slovakia & 1 \\
\hline \multirow[t]{2}{*}{ Islamic Republic of Iran } & 13 & Zambia & 1 \\
\hline & & Total & 10589 \\
\hline
\end{tabular}

stitute and Partners in Health, including the Millennium Villages Project (MVP), Jembi Health Systems and eHealth Systems. An OpenMRS Centre of Excellence $\mathrm{CoE})$ refers to an organization established to assist with OpenMRS development and by the CoE developers who assist with local customization and also contribute to the core development of OpenMRS.

\section{Community Outreach}

The OIN has grown and reached beyond its own network boundaries to embrace like-minded structures and approaches working toward the same goal of improve health outcomes through better use of information throughout the health system. Some of these nodes to which OIN has reached are described below.

\section{The Millennium Villages Project}

MVP operates in 14 clusters of villages in 10 sub-Saharan African countries and hopes to help villages achieve the Millennium Development Goals (MDGs) through the implementation of comprehensive, community-based, low-cost, integrated rural development strategies including primary health care. MVP is a partnership between the Earth Institute, Columbia University, the United Nations Development Program, Millennium Promise (an NGO) and the governments of the African countries.

The Millennium Global Village-Network (MGV-Net) is a health information and communication network designed for MVP communities that is built upon OpenMRS and other open source information and communication technologies. MGV-Net requires human resource capacity at all levels of the health information chain from the community all the way to the government. Working with support from Rockefeller Foundation, the International Development Research Centre, the Novartis Fund for Sustainable Development and Ericsson, MVP needs to train and equip a cadre of implementers and educated users in multiple countries and in multiple languages. Within each MVP country, MVP is training an eHealth Specialist who can then train the necessary data entry clerks, analysts and care providers to take advantage of the new technologies. MVP is also working at the policy level to help 
decision-makers implement and take advantage of ICT for health.

\section{Jembi Health Systems}

Jembi Health Systems is developing, implementing and supporting systems in several African countries, including South Africa, Zimbabwe, Mozambique and Sierra Leone. Jembi also hosts the annual OpenMRS Implementers Meeting and has partnerships with several other open source software developer and implementer networks. Jembi developers have excelled in OpenMRS development and implementation and also specialize in interoperability having implemented FOSS interoperability laboratories (FOSSIL) in Cape Town and Durban. Notable achievements include the development of a Java library for SDMX-HD, integration of OpenMRS with the DHIS and mentoring assignments as part of the Google Summer of Code programme. Jembi developers have also been developing code under contract to other members of the OpenMRS consortium, particularly developing interoperable functionality between OpenMRS and other open source applications.

\section{eHealth Systems}

eHealth Systems (eHS) is a Chilean company that provides health information technology (IT) consulting and system implementation to clinical organizations, especially in the public sector. Currently, eHS is working with Clinica Santa Maria, one of the largest clinics in Chile, developing OpenMRS for the Chilean primary care system, and co-organizing, for the second time, the Open Source Health Informatics Conference for Latin America and the Caribbean (IMeCA; www.informaticamedica-lac.org) with the Sustainable Sciences Institute.

\section{Discussion}

Free and open source software (FOSS) is an important alternative for information systems development in the health domain, particularly in developing coun- tries [18]. A thriving medical open source developer community exists [19] and it is important to understand the factors leading to ongoing success and sustainability of these communities, if the software is to be used. Strong developer and implementer communities are one of the most important features of successful FOSS applications, resulting in better growth and sustainability of the applications and also fostering learning within the community [20].

Several theories exist as to what motivates open source developers and implementers. In addition to personal enjoyment, it appears that interpersonal relationships and leadership effectiveness are important factors in attracting volunteer developer involvement in open source development projects [21]. Furthermore, open source project members are also influenced by the community environment, and the quality of an open source project community was found to be important in promoting the involvement of project members [21]. Kimaro et al [22] have argued that extra effort needs to be put into mentoring and training intended users from low income countries. The implementation process satisfies their criteria for participatory customization, explaining why the implementer network is relatively successful, by comparison.

The success of OpenMRS and the role of the OIN can also be interpreted in terms of complexity theory. According to this theoretical framework, OpenMRS is considered to be a complex adaptive system (CAS) comprising a number of components with complex interactions between them and attributes such as self-organization and co-evolution $(23,24)$. The OIN is considered to be a 'complex adaptive agent' within the overall system that has co-evolved with other elements such as the developer group and the software itself. The large growth of the OIN has resulted from a relatively simple intervention, ie the formation of a dedicated list server and holding an annual meeting which is interpreted as an example of the nonlinear behaviour of CAS. In addition, there is relatively little supervision of this function, illustrating the self-organizing property of the OIN as a CAS.

Co-evolution is another important attribute of CAS. The developer and implementer networks are tightlybound to one another and have evolved attributes of the other. Many implementers have become developers and developers have become implementers. The implementer groups that started in Africa, Latin America and other resource-constrained settings have evolved to take on attributes that are more characteristic of developer groups. In addition, the OIN Annual Meeting has evolved from a traditional user-group style into an "unconferencing" style, characteristic of developer communities, complete with hackathons, codefests and interoperability connectathons, catering for both the developer and implementer communities.

The OIN has had an important influence on the development and growth of the OpenMRS software itself and probably contributed significantly to its success by helping to keep it focused and relevant. The OpenMRS community is highly distributed and geographically dispersed and the OIN is an important conduit for information about the important implementation constituency to the leadership and developer groups, allowing them to coevolve and adapt to changing circumstances, further strengthening the network. The OIN has also introduced other open source communities to the developer group as part of the OIN Annual Meeting, contributing to further evolution and refinement of the network. Through active collaboration with groups providing related applications, OpenMRS has been able to focus on its core mission of becoming an excellent EMR while simultaneously satisfying requirements for enterprise scale solutions. Formal and informal links have been formed with these other self-organizing communities and networks of networks.

Community-driven approaches are more likely to achieve the benefits ex- 
pected from open source projects, including the following:

- Greater range of functionality and quality of code. This is directly proportional to the number of developers contributing to the code base and modules.

- Improved Sustainability. FOSS applications without strong community support will naturally struggle to reach the level of functionality provided by commercial applications and will also be more risky in terms of what can be achieved.

- Improved enterprise-readiness. A strong code base and community are necessary components of FOSS that is considered for adoption for mission-critical applications. However, they are not sufficient for many enterprise applications due to the fact that they are restricted to one domain. Development of interoperability with other open source applications, such as that between OpenMRS and DHIS, iDART and JavaROSA, increases the range of functionality and applications of the combined whole, resulting in greater application at the enterprise level.

\section{Conclusions}

The OpenMRS Implementers Network has made several important contributions to knowledge of how FOSS is applied. In particular, it has demonstrated one way to grow an open source community to assist with the important aspects of FOSS implementation and support that are not as well developed in the open source community as is FOSS development.

The OpenMRS Implementers Network has made a significant contribution to the development of the human resource capacity and the software that serves a growing number of communities all over the world. Building a health information system that is scalable, sustainable and strengthens existing health care systems requires a large number of trained and motivated people. When combined with interoperable, scalable and customizable software, this informatics-enabled workforce will play a major role in delivering more and better health care for more and more people.

\section{Acknowledgements}

The OpenMRS Implementers Network is partially supported by a grant from the Canadian International Development Research Centre (IDRC) ICT4D program: "Developer Network and Open Source PDA Software for Health Data Collection" (Grant Number 101974001) with additional inputs from the South African Medical Research Council (SA-MRC), the World Health Organization and the Rockefeller Foundation. PIH is supported by WHO and the Regenstrief Institute is supported by the US Centres for Disease Control. The authors gratefully acknowledge ongoing support and contributions from Heloise Emdon, Chaitali Sinha and Steve Song, IDRC and Prof Bill Tierney, Regenstrief Institute and University of Indiana.

\section{References}

1. AbouZhar C, Boerma T. Health information systems: the foundations of public health. Bulletin of the World Health Organisation, (83:8); 2005. p. 578-83.

2. Bukachi F, Pakenham-Walsh N. Information technology for health in developing countries. Chest 2007 Nov;132(5):1624-30.

3. Titlestad OH, Staring K, Braa J. Distributing Development to Enable User Participation: Multilevel Design in the HISP Network, Scandinavian Journal of Information Systems 2009;21(1):27-50.

4. Levison L, Fraser HSF. Requirements for an OpenSource Pharmacy Dispensing and Stores Management Software Application for Developing Countries, Paper presented at Making the eHealth Connection Conference, Bellagio, Italy, July 13 - Aug. 8, 2008 edn.

5. Mamlin BW, Biondich PG, Wolfe BA, Fraser H, Jazayeri D, Allen C, et al. Cooking up an open source EMR for developing countries: OpenMRS a recipe for successful collaboration. AMIA Annu Symp Proc 2006:529-33

6. Mamlin BW, Biondich PG. AMPATH Medical Record System (AMRS): collaborating toward an EMR for developing countries. AMIA Annu Symp Proc 2005:490-4

7. Tierney WM, Rotich JK, Hannan TJ, Siika AM, Biondich PG, Mamlin BW, et al. The AMPATH medical record system: creating, implementing, and sustaining an electronic medical record system to support HIV/AIDS care in western Kenya. Stud Health Technol Inform 2007;129(Pt 1):372-6.

8. The Open Architectures, Standards and Information Systems Project. IDRC; www.idrc.ca/en/ev116782-201-1-DO_TOPIC.html

9. Seebregts CJ, Mamlin BW, Biondich PG, Fraser HS, Wolfe BA, Jazayeri D, et al. The OpenMRS Implementers Network. Int J Med Inform 2009 Nov; 78(11):711-20.

10. Allen C, Manyika, P, Jazayeri D, Rich M, Lesh N, Fraser H. Rapid deployment of electronic medical records for ARV rollout in rural Rwanda. Proc AMIA
Symp 2006:840.

11. Allen C, Jazayeri D, Miranda J, Biondich PG, Mamlin BW, Wolfe BA, et al. Experience in implementing the OpenMRS medical record system to support HIV treatment in Rwanda. Stud Health Technol Inform 2007;129(Pt 1):382-6.

12. Kanter AS, Negin J, Olayo B, Bukachi F, Johnson E, Sachs SE. Millennium Global Village-Net: Bringing together Millennium Villages throughout sub-Saharan Africa. Int J Med Inform 2009 Sep 17.

13. Douglas G, Gadabu O, Joukes S, Mumba S, McKay $\mathrm{MV}$, et al. Using touchscreen electronic medical record systems to support and monitor national scale-up of antiretroviral therapy in Malawi. PloS (accepted)

14. Seymour RP, Tang A, DeRiggi J, Munyaburanga C, Cuckovitch R, Nyirishema P, Fraser HSF Training Software Developers for Electronic Medical Records in Rwanda, Proc Med Info2010 (in press)

15. Braa J, Hedberg C. The struggle for district-based health information systems in South Africa. Inf Soc 2002;18(2):113-27.

16. Braa J, Monteiro E, Sahay S. Networks of action: Sustainable health information systems across developing countries. MIS Quarterly: Management Information Systems 2004:28(3):337-62.

17. Braa J, Kanter AS, Lesh N, Crichton R, Jolliffe R, Seebregts CJ. Comprehensive Yet Scalable Health Information Systems for Low Resource Settings: A Collaborative Effort in Sierra Leone. Submitted.

18. Fraser HSF, Biondich P, Moodley D, Choi S, Mamlin BW, Szolovits P. Implementing electronic medical record systems in developing countries, Inform Primary Care 2005;13:83-95.

19. Janamanchia B, Katsamakas E, Raghupathi W, Gao W. The State and Profile of Open Source Software Projects in health and medical informatics. International J Med Inform2009;78:457-72.

20. Lakhani KR, von Hippel E. How open source software works: "free" user-to-user assistance. Research Policy 2003;32(6):923-43

21. Xu B, Jones DR, Shao B. Volunteers' involvement in online community based software development. Information \& Management 2009; 46:151-8

22. Kimaro HC, Titlestad $\mathrm{OH}$. Challenges of user participation in the design of a computer based system: the possibility of participatory customisation in low income countries [Electronic Version]. Journal of Health Informatics in Developing Countries 2008:2,1-9 from http:// www.jhidc.org/index.php/jhidc/issue/view/4.

23. Jacucci EO, Hanseth et al. Introduction - taking complexity seriously in IS research. Information Technology and People 2006;19(1): 5-11.

24. Shaw V. A Complexity Inspired Approach to CoEvolutionery Hospital Management Information Systems Development. Case Studies from the "South". (2009). PhD Thesis, University of Oslo.

\section{Corespondence to:}

Christopher I Seebregts

Biomedical Informatics Research

eHealth Research and Innovation Platform

Medical Research Council

PO Box 19070, Tygerberg 7505

South Africa

Tel.: + 27219380318

Fax: + 27219380526

E-mail: chris.seebregts@mrc.ac.za 